\title{
Long-range energy transfer in proteins
}

\author{
Francesco Piazza $^{1}$ and Yves-Henri Sanejouand ${ }^{2}$ \\ ${ }^{1}$ Ecole Polytechnique Fédérale de Lausanne, Laboratoire de Biophysique Statistique, ITP-SB, BSP-720, \\ CH-1015 Lausanne, Switzerland \\ ${ }^{2}$ Laboratoire Biotechnologie, Biocatalyse et Biorégulation, UMR 6204 du CNRS, Faculté des Sciences \\ et des Techniques, 2, rue de la Houssinière, 44322 Nantes Cedex 3, France \\ E-mail: francesco.piazza@epfl.ch and Yves-Henri.Sanejouand@univ-nantes.fr
}

Received 19 August 2009

Accepted for publication 20 October 2009

Published 12 November 2009

Online at stacks.iop.org/PhysBio/6/046014

\begin{abstract}
Proteins are large and complex molecular machines. In order to perform their function, most of them need energy, e.g. either in the form of a photon, as in the case of the visual pigment rhodopsin, or through the breaking of a chemical bond, as in the presence of adenosine triphosphate (ATP). Such energy, in turn, has to be transmitted to specific locations, often several tens of $\AA$ away from where it is initially released. Here we show, within the framework of a coarse-grained nonlinear network model, that energy in a protein can jump from site to site with high yields, covering in many instances remarkably large distances. Following single-site excitations, few specific sites are targeted, systematically within the stiffest regions. Such energy transfers mark the spontaneous formation of a localized mode of nonlinear origin at the destination site, which acts as an efficient energy-accumulating center. Interestingly, yields are found to be optimum for excitation energies in the range of biologically relevant ones.
\end{abstract}

\section{Introduction}

Protein dynamics is encoded in their structures and is often critical for their function [1]. Since the early 1980s, it is well known that vibrational non-harmonicity [2, 3] has to be accounted for to understand intra-structure energy redistribution [4-8]. Among nonlinear effects, long-lived, localized modes were suggested to play a key role both by theoretical [9-11] and experimental studies [12-15]. For example, localized vibrations in $\alpha$-helices have been recently hypothesized to represent a means of concentrating energy during enzymatic catalysis [16, 17]. More generally, persistent motions in hinge regions have been shown to correlate with enzymatic activity by NMR measurements [18], thus prompting the very idea that some kind of energy storage is required at the pico- to nano-second time scale to regulate functional motions at much longer time scales typical of protein function.

Possible modes of nonlinear origin suggested to influence protein dynamics include topological excitations, such as solitons [19, 20], as well as Discrete Breathers (DB) [21, 22]. The latter, also known as intrinsic localized modes (ILMs), are spatially localized, time-periodic vibrations found generically in many-body systems as a combined effect of nonlinearity and spatial discreteness [23, 24]. Their existence and stability properties have been widely explored in translationally invariant systems [25] at $T=0$, and much effort has also been devoted to understanding the role of thermal noise on DBs [26-29]. However, little is known on how DBs are affected by the interplay of spatial heterogeneity and nonlinearity $[11,30,31]$, a fortiori in the context of atomic fluctuations in biological macro-molecules.

A number of general features make discrete breathers appealing candidates for playing a role in energy storage and circulation processes in proteins. Notably, DBs are able to harvest from the background and pin down for long times amounts of energy much larger than $k_{B} T$. Indeed, their ability to pump energy from neighboring sites is a distinctive signature of DB self-excitation [32], e.g. observed as a consequence of surface cooling [33-36] or due to modulational instability of band-edge waves in nonlinear lattices [37, 38]. Furthermore, within the frame of a nonlinear network model, we have shown that highly energetic DBs can form easily in proteins [33], and that they feature strongly site-modulated properties [39]. More precisely, we have shown that spatially localized bandedge normal modes (NM) can be continued from low energies to DB solutions centered at the same sites as the corresponding NMs (the NM sites). Note that the latter lie, as a rule, within the stiffest regions of a protein [33, 39]. More generally, however, DBs display a gap in their excitation spectrum. As a 
consequence, they can 'jump' to another site as their energy is varied, following spatial selection rules matching the pattern of DBs localized elsewhere [39]. As a matter of fact, such jumps realize efficient energy transfers. Hereafter, we show that events of this kind, connecting with high yields even widely separated locations, can be triggered by a localized excitation, so long as its energy $E_{0}$ lies above a given threshold. Indeed, provided that transfer phenomena of this kind are compatible with cellular constraints, it is tempting to speculate that evolution has found a way to put such long-lived modes at work for lowering energy barriers associated with chemical reactions, e.g. for boosting enzyme efficiency during catalytic processes [40].

\section{Methods}

Proteins are modeled as networks of nodes of mass $M$ (the $\alpha$-carbons of their amino acid residues) linked by springs. Specifically, in the nonlinear network model (NNM) [33, 39], the potential energy of a protein, $E_{p}$, has the following form:

$$
E_{p}=\sum_{d_{i j}^{0}<R_{c}}\left[\frac{k_{2}}{2}\left(d_{i j}-d_{i j}^{0}\right)^{2}+\frac{k_{4}}{4}\left(d_{i j}-d_{i j}^{0}\right)^{4}\right]
$$

where $d_{i j}$ is the distance between particles $i$ and $j, d_{i j}^{0}$ their distance in the equilibrium structure (as e.g. solved through x-ray crystallography) and $R_{c}$ is a distance cutoff that specifies which pairs of nodes are interacting. Note that $k_{4}=0$ corresponds to the widely used elastic network model (ENM) [41-43], which has proven useful for quantitatively describing amino acid fluctuations at room temperature [41, 42, 44, 45], as well as for predicting or characterizing large-amplitude functional motions of proteins [46-50] in agreement with all-atom models [51-53], paving the way for numerous applications in structural biology [54-57].

As in previous NNM studies [33, 39], we take $R_{c}=$ $10 \AA, k_{4}=5 \mathrm{kcal} / \mathrm{mol} / \AA^{4}$ and fix $k_{2}$ so that the low-frequency part of the linear spectrum matches actual protein frequencies, as calculated using realistic force fields [54]. When $M=$ $110 \mathrm{amu}$ (the average amino acid residue mass), this gives $k_{2}=$ $5 \mathrm{kcal} / \mathrm{mol} / \AA^{2}$. Note that, in view of their coarse-grained nature, network models do not reproduce the high-frequency side of an all-atom linear spectrum. Normal modes in this region correspond to localized motions of chemically bonded atoms that are not included in the model. Moreover, frequencies of these motions are so high (up to $\approx 4000 \mathrm{~cm}^{-1}$ $[58,59])$ that, as a consequence of quantum-mechanical effects, most of them are unlikely to be excited at room temperature $\left(v>k_{B} T / h \approx 200 \mathrm{~cm}^{-1}\right)$. Note also that choosing a given value of $k_{4}$ amounts to define a typical distance $\ell=\sqrt{k_{2} / k_{4}}$ above which nonlinearity starts affecting significantly atomic fluctuations $(\ell=1 \AA$ in our case, meaning rather weak anharmonicity). However, it is important to stress that in our dynamical system, energy and $k_{4}$ are not independent parameters. Therefore, performing an energydependent study at fixed $k_{4}$ or acting vice versa will not yield qualitatively different results. In fact, a simple rescaling of the appropriate units (energies or lengths) would suffice to map ones onto the others.

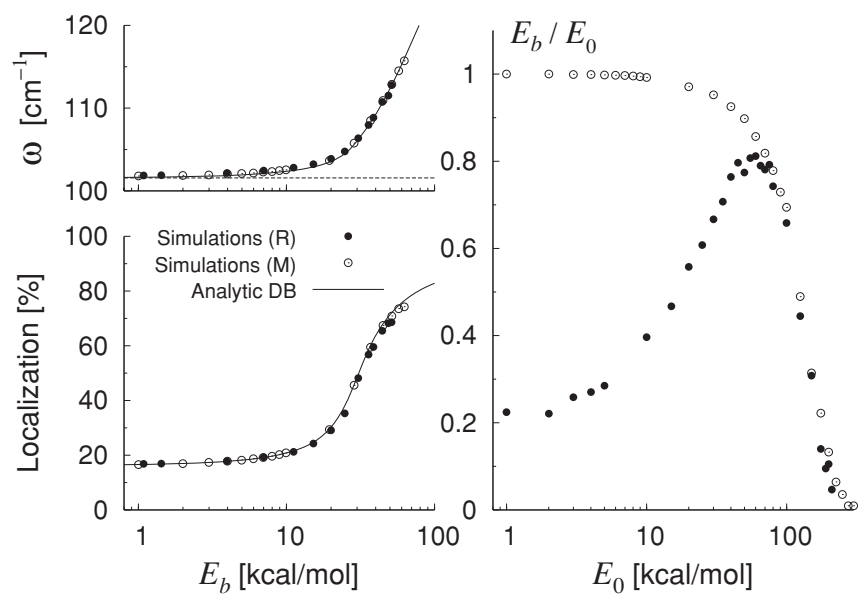

Figure 1. Local kicks cause energy pinning through the excitation of discrete breathers. The figure illustrates the formation of a discrete breather at site VAL 177 in subtilisin (PDB code 1AV7), a 274-amino-acid enzyme, following the excitation of the band-edge normal mode (M) or a single-site kinetic energy kick at VAL 177 $(\mathrm{R})$, the residue where the edge NM is centered. The right panel reports the energy $E_{b}$ found in the nonlinear localized mode as a function of the excitation energy $E_{0}$. The left panels compare the frequency $\omega$ and the localization index $L$ of the nonlinear mode with those of discrete breather solutions centered at VAL 177 calculated analytically, as described in [39], showing that the nonlinear mode excited after a kick is indeed a discrete breather. The dashed line in the upper-left plot marks the band-edge frequency of the protein network $\left(v_{E}=101.6 \mathrm{~cm}^{-1}\right)$.

For each site in a given structure, the maximum-stiffness (MS) direction is computed through the sequential maximum strain algorithm [39]. Following an initial kinetic-energy impulse (kick) at a specific site along the local MS unit vector, a 2 ns microcanonical simulation is performed. After a $1 \mathrm{~ns}$ transient period during which a part of the excitation energy flows into the system, the velocity-covariance matrix is computed. Its first eigenvector provides the pattern of correlated site velocities involved in the dominant (most energetic) nonlinear mode (the DB). Accordingly, a transfer is recorded at the site at which this mode is found localized. Projecting the system trajectory on the same eigenvector yields fair estimates of the DB frequency and average energy [33].

The localization index $L$ of a DB centered at site $m$ is obtained from the weight of the latter in the normalized displacement pattern of the DB, namely $L=100 \times$ $\sum_{\alpha=x, y, z}\left[\xi_{\alpha}(m)\right]^{2}$, where $\xi_{\alpha}(m)$ are the components at site $m$ of the corresponding eigenvector.

\section{Results}

\subsection{Discrete breather excitation}

Figure 1 summarizes the outcome of one such experiment, where energy is initially either localized in NM (M) or in real $(\mathrm{R})$ space. Typically, the initial excitation is found to spark the formation of a discrete breather, pinning a variable amount of energy $E_{b}$ at a specific location. When less than $10 \mathrm{kcal} \mathrm{mol}^{-1}$ of kinetic energy is injected into the edge NM, 


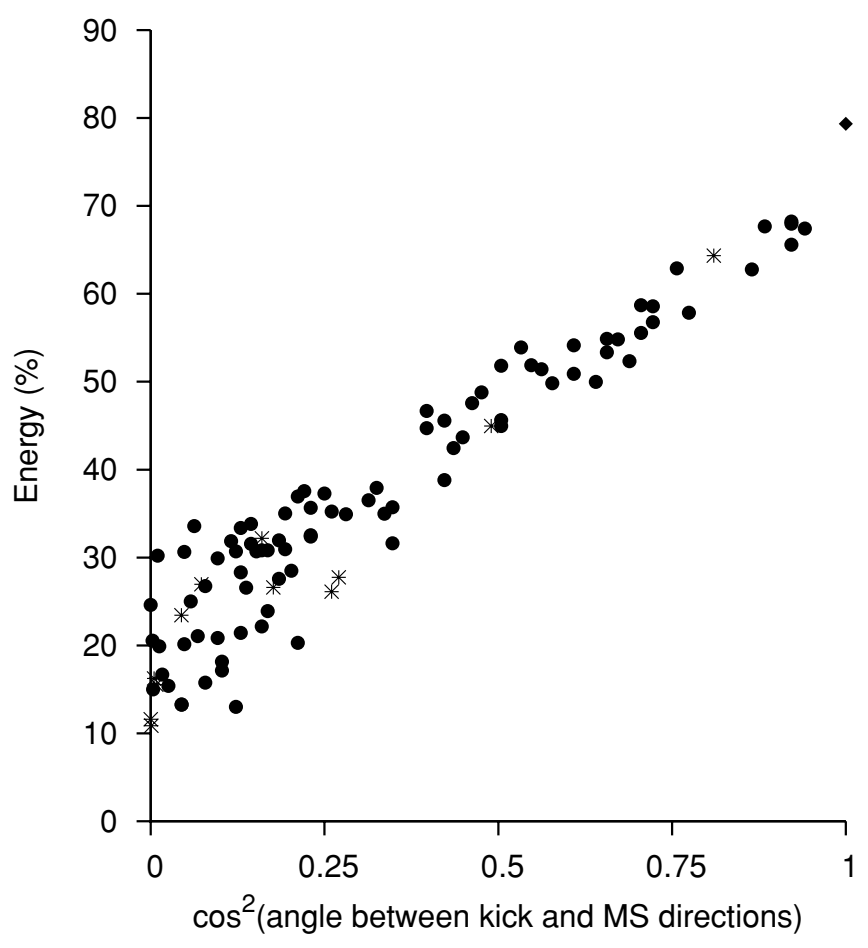

Figure 2. Optimum kick direction for exciting discrete breathers in dimeric citrate synthase (PDB code 1IXE). Percentage of the system energy found in a nonlinear mode as a function of the direction of the initial kick given to SER 213A, the NM site of the band-edge mode. The latter is measured by the angle $\theta$ between the kick direction and the MS unit vector. In all simulations, the (kinetic) energy of the kick is $55 \mathrm{kcal} \mathrm{mol}^{-1}$ and its direction is chosen at random, except that when the maximum strain (MS) direction is picked instead (black diamond at $\cos \theta=1$ ). Filled circles: SER $213 \mathrm{~A}$ is found to be the most energetic site during the analysis timespan. Stars: it is another one. In one instance, while the kick was given in a direction close to the MS direction $(\cos \theta=0.9)$, the DB jumped on a neighboring site (namely, THR 208A).

nearly all this energy is kept by the DB, whose overlap with the edge $\mathrm{NM}$ is large at low energies. Increasing $E_{0}$ further, the frequency of the excited mode detaches from the linear band, while the excitation efficiency $E_{b} / E_{0}$ is eroded. In fact, as DB localization builds up with energy (see the lower-left panel), the spatial overlap with the edge NM diminishes, thus reducing excitation efficiency [4]. The same DB is also excited when the edge NM site is 'kicked' along an appropriate direction, namely the MS one [39] (see data marked (R) in figure 1). In this case, however, the excitation becomes more efficient as $E_{0}$ is increased since the DB asymptotically approaches a single-site vibration. For $E_{0}>100 \mathrm{kcal} \mathrm{mol}^{-1}$, the DB loses its energy, which flows rapidly into the system.

\subsection{Directional specificity}

We find that the MS direction invariably allows for the most efficient excitation of a nonlinear mode at a given site. Figure 2 illustrates the efficiency of kicks given along the MS direction, with respect to kicks imparted along random directions. The correlation with the squared cosine of the angle between the kick and the MS unit vectors indicates that it is the

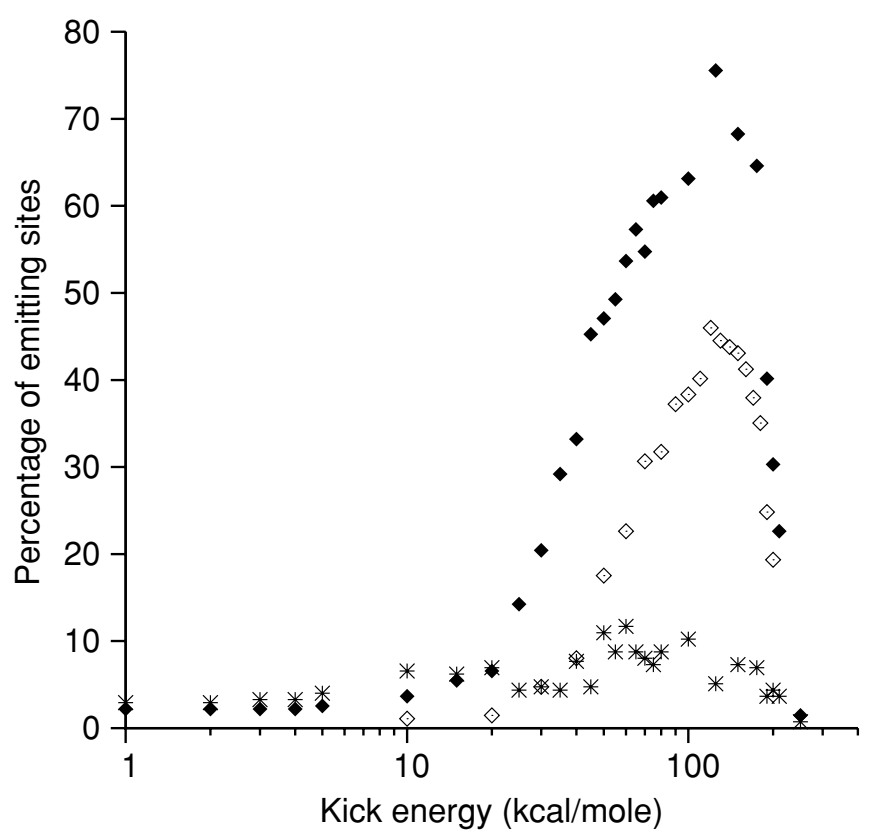

Figure 3. Energy transfer: all-site analysis. Percentage of sites in subtilisin that transmit most of the kick energy to the band-edge NM site, VAL 177 (black diamonds), or to the NM site of the second edge mode, MET 199 (stars). For a given kick energy, each site is kicked once, the most energetic nonlinear mode obtained is analyzed and the site the most involved in this mode is recorded. When initial excitations are not imparted along the local stiffest direction, but are oriented at random, energy transfer toward VAL 177 is less likely (open diamonds).

amount of energy injected along the MS vector which is the dominant factor allowing for efficient excitation of a discrete breather.

Interestingly, kicking away from the MS direction can promote energy transfer to another site. For instance, while a kick along the MS unit vector at the NM site of the band-edge mode invariably results in a DB sitting at the same site, when the direction of the kick is picked at random, discrete breathers localized elsewhere are also observed (see again figure 2). In the following, we take advantage of the fact that MS directions can be easily calculated at any site in any structure [39] in order to investigate energy transfer in a systematic manner.

\subsection{Energy transfer}

When a given residue is kicked along the MS direction, a transfer event can occur when $E_{0}>\approx 20 \mathrm{kcal} \mathrm{mol}^{-1}$ (see an example in figure 3). At peak transfer, more than $75 \%$ of such kicks excite a DB localized at the band-edge NM site, while otherwise energy flows toward the NM site of another edge mode. Conversely, when the kick is imparted along a random direction, energy transfer is found to be less efficient.

Quite generally, a transfer event can be observed when almost any site is kicked, and in the majority of cases only a handful of well-defined sites are targeted. This means that energy transfer can occur between widely separated locations. Indeed, as illustrated in figure 4 for myosin, only about $5 \%$ of $55 \mathrm{kcal} \mathrm{mol}^{-1}$ kicks result in a DB localized at the same 


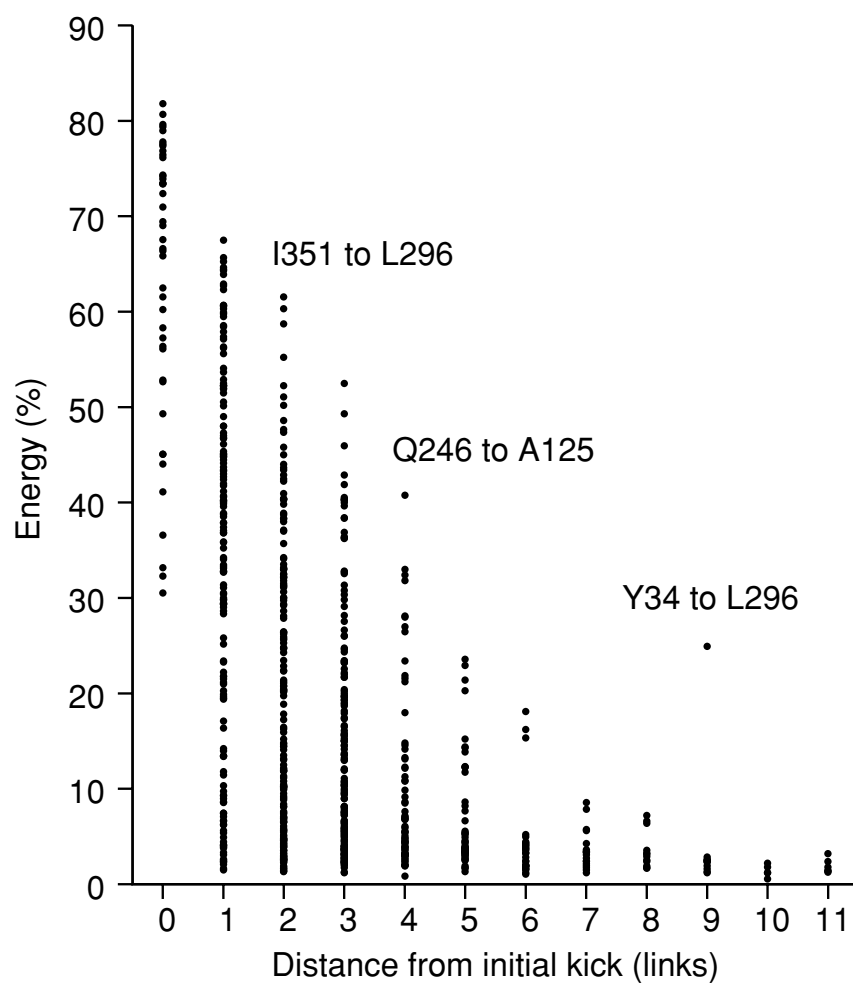

Figure 4. Energy transfer as a function of distance from the excitation site. The figure illustrates the outcome of an all-site kick experiment in myosin, a large 746-amino-acid enzyme involved in muscle contraction (PDB code 1VOM). The fraction of excitation energy found in the DB is plotted versus the distance (in units of links in the connectivity graph) between the kicked site and the site where the nonlinear mode self-excites. The maximum amount of energy found in the DB decreases with the number of links separating the feed and the target sites. For instance, when GLN 246 is kicked, more than $40 \%$ of the energy ends up in a DB localized at ALA 125 (the band-edge NM site). This amounts to four links, corresponding to a span of about $25 \AA$ in real space. Otherwise, when a kick is given to ILE 351 or TYR $34,25-65 \%$ of the excitation energy flows either to ALA 125 or LEU 296, the NM site of the third edge normal mode. In cases where more than $30 \%$ of the kick energy is transferred away, three sites turn out to be targeted half of the times, namely ALA 125 (27\%), LEU 296 (13\%) and GLY $451(7 \%)$. When only long-range energy transfers are considered (covering three or more links), the shares raise to $71 \%$ and 18\% for ALA 125 and LEU 296, respectively. In the remaining cases, the DB is found either at LEU $516(7 \%, 14$ th mode) or at ARG 80 (4\%, 10th mode).

location. For all other kicked sites, a transfer occurs to a DB pinning a decreasing fraction of the excitation energy, 1 to 11 links away. Note that all high-yield and long-range energy transfers aim at the NM sites of one of the edge NMs, the NM site of the bande-edge mode being the most likely target. Thus, energy systematically flows toward the stiffest regions of the structure. Interestingly, this is where functionally relevant residues tend to be located [33, 39, 60-62].

In one occurrence, more than $20 \%$ of the kick energy ends up in a nonlinear mode localized more than five links away: following a kick at TYR 34, a remarkable nine-link stretch is covered up to LEU 296, making a jump of more than $60 \AA$. However, cases of ultra-long-range energy transfer like this

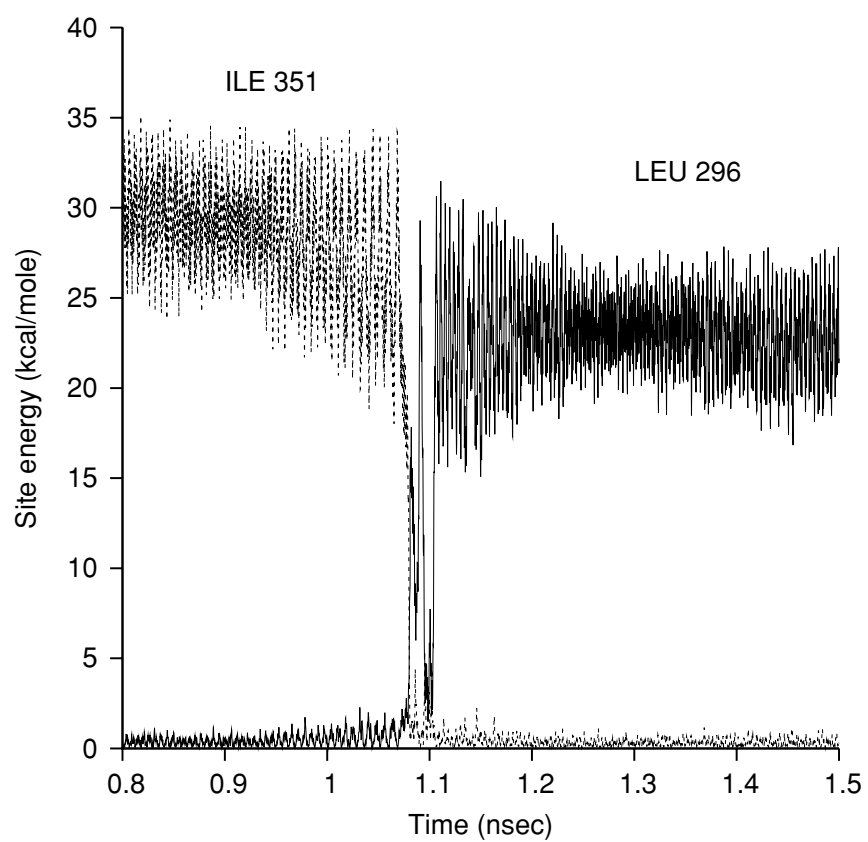

Figure 5. Site-to-site energy transfer in myosin. The local energies at sites ILE 351 (dotted line) and LEU 296 (solid line) are plotted as functions of time, after a $55 \mathrm{kcal} \mathrm{mol}^{-1} \mathrm{kick}$ at ILE 351 . The fluctuations occurring well before and after the transfer reflect the fact that the corresponding nonlinear modes are not perfectly localized on both sites. As a consequence, they exchange significant amounts of energy with their environs.

are more rare and, at the same time, less efficient. In fact, as a consequence of the rather small amount of energy transferred (nearly $14 \mathrm{kcal} \mathrm{mol}^{-1}$ ), the DB that self-excites at the target site is poorly localized (as in figure 1).

A more efficient transfer event, covering two links (about $11 \AA$ ), is analyzed in figure 5. At first, a DB is excited at the kicked site. However, due to interactions with the background, its energy slowly but steadily flows into the system. After approximately $1 \mathrm{~ns}$, about $65 \%$ of the excitation energy is still there. At $t=1.1 \mathrm{~ns}$, this amount of energy is rapidly and almost entirely transferred to LEU 296, marking the selflocalization of another DB. Although the transfer itself is a quite complex process, involving several intermediate sites, it may well prove to be an example of targeted energy transfer [21]. Indeed, as the energy of the DB at the initial site drops, its frequency diminishes as well. This may allow for a transfer to occur if a resonance condition with the frequency of another DB is met. The transmission should be irreversible, as a consequence of both DBs' frequency drifts during energy exchange [21]. Note that, as the energy of the first DB is eroded, the mode becomes also less and less localized [39]. This, in turn, is likely to increase the overlap between the two DB displacement patterns, thus allowing for more efficient energy channeling $[4,8]$.

To gain further understanding on the transfer mechanism, we investigated energy circulation in a dimeric form of rhodopsin. Very few high-yield and long-range energy transfers were recorded between sites belonging to different monomers, the vast majority of transfer events being confined within the same domain. Indeed, in less than $1 \%$ of the 


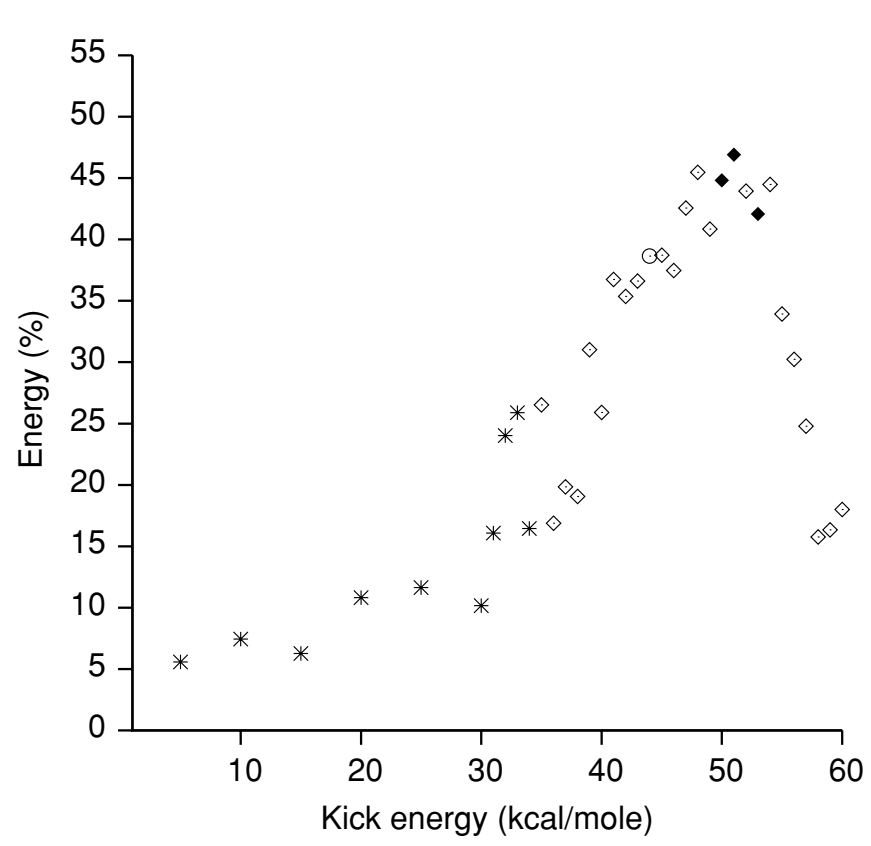

Figure 6. Energy transfer in rhodopsin (PDB code 3CAP). The fraction of energy $E_{b} / E_{0}$ found in the discrete breather when kicking the site attached to the retinal chromophore (LYS 296) of monomer B is plotted versus the excitation energy. Symbols indicate at which site the DB self-localizes: GLU 113 (black diamonds), CYS 185 (open diamonds), MET 86 (open circle) or another one (stars).

instances more than $30 \%$ of the kick energy $\left(55 \mathrm{kcal} \mathrm{mol}^{-1}\right)$ injected at one monomer is transmitted to the other. Here, at variance with most protein dimers, the stiffest regions are located in monomer bulks, so that the edge NMs are localized far away from the interface. This strongly suggests that energy transfers not only target stiff regions, but can also couple any two sites efficiently only through rather stiff channeling pathways. On the other hand, when kicking one of the two (almost) equivalent sites of rhodopsin that are covalently linked to the retinal chromophore, up to about $50 \%$ of the excitation energy ends up in a DB localized at one of three specific sites, the targeted location depending upon where (which monomer) the kick is imparted and on the magnitude of the latter. Interestingly, figure 6 reveals that transfer efficiency is optimum in the narrow range $50-55 \mathrm{kcal} \mathrm{mole}^{-1}$, i.e. exactly the energy of photons that can be absorbed by the retinal chromophore when it is embedded within rhodopsin $(\lambda=500 \div 550 \mathrm{~nm})$. Moreover, the preferentially targeted residue in this energy range (GLU 113) is known to be involved in the early stages of the signaling cascade following rhodopsin activation [63].

\section{Discussion}

In summary, despite its coarse-grained nature, the NNM framework is able to provide biologically sensible clues about energy circulation in proteins. High-yield and long-range energy transfers systematically pin energy at the sites the most involved in a small subset of band-edge linear modes, that is, within the stiffest parts of protein structures. These, in turn, are regions preferentially hosting residues involved in catalytic mechanisms [33, 39, 60-62].

In our previous works, the fact that DBs tend to form within the most rigid parts of protein structures had already been underlined. First, by showing that this is where they spontaneously pop up in the course of simulations during which a protein is cooled down as a consequence of friction on its surface residues [33]. Second, by showing that this is where $\mathrm{DB}$ analytical solutions can be continued down to arbitrary small energies [39]. Importantly, the latter study also allowed us to show that DBs are able to jump from site to site following energy variations. The present results reveal how efficient this property can be for transmitting energy across protein structures, so that a few key residues are fired at following localized excitations. As a clear example, figure 3 shows that a significant fraction of the energy of a $100 \mathrm{kcal} \mathrm{mol}^{-1} \mathrm{kick}$ in subtilisin ends up on a single target residue, the stiffest one, for almost every kicked site. Recalling again that active sites have a marked tendency to sit within the stiffest regions [61, 62], what our results suggest is that protein structures may have been designed, during the course of evolution, so as to allow energy to flow where it is needed, e.g. to, or close to catalytic sites. Such extra amounts of energy, with respect to what is commonly available at room temperature, may help crossing rate-limiting energy barriers, thus speeding up catalytic processes by a significant factor. Incidentally, this conclusion is in line with the phylogenetic analysis of structural evolution within protein families, clearly spotlighting the existence of clusters of coevolving amino acids [64, 65], which may prove associated with preferential energy exchange pathways. Note that such energy transfer processes do not have to be directly linked to conformational changes occurring on the ms time scale, in line with recent results that challenge the very coupling between conformational and chemical transitions in enzymes [66].

However, intensive work remain to be done in order to confirm the above appealing hypothesis. For instance, in order to bridge the gap between the nanosecond time scale of the nonlinear phenomena studied herein and micro to millisecond time scales, which are typical of biologically relevant events occurring within enzymes, DB lifetimes have to be accurately examined, noteworthy as a function of their energy. To this concern, it is interesting to recall that, as a general effect, friction exerted by solvent at the protein surface results in an overall stiffening of the protein bulk [33]. Hence, it is likely, even if somewhat paradoxical, that the lifetimes of DBs harbored in stiff regions could be increased by solvent's action. Work along this line is in progress.

Of course, demonstrating that DBs are among the key players of enzyme catalysis would also require to consider much more detailed protein models. Indeed, in the framework of NNMs, DBs arise as high-frequency motions of the coarsegrained network. Consequently, since in proteins highfrequency vibrations are localized on chemical bonds and hence behave quantum-mechanically, a semi-classical or even full quantum-mechanical description may prove necessary. Moreover, while we have already shown that DBs are able to arise in the presence of friction acting on nearly half of 
the amino acids (those lying on its surface) [33], it is now important to assess how DB properties are influenced by the Brownian noise component of surface forces due to solvent.

As an end remark, we note that, in view of the coarsegrained nature of the NNM scheme, the same site-specific, high-yield and long-range energy transfers observed herein in the case of proteins are also likely to occur in other physical systems, possibly simpler to engineer and to handle, so long as they share with proteins both spatial and stiffness heterogeneity.

\section{References}

[1] Karplus M and Kuriyan J 2005 Proc. Natl Acad. Sci. USA 102 6679-85

[2] Levy R, Perahia D and Karplus M 1982 Proc. Natl Acad. Sci. USA 79 1346-50

[3] Hayward S and Go N 1995 Annu. Rev. Phys. Chem. $46223-50$

[4] Moritsugu K, Miyashita O and Kidera A 2000 Phys. Rev. Lett. 85 3970-3

[5] Sagnella D, Straub J and Thirumalai D 2000 J. Chem. Phys. $1137702-11$

[6] Hennig D 2002 Phys. Rev. B 65174302

[7] Ishikura T and Yamato T 2006 Chem. Phys. Lett. 432 533-7

[8] Leitner D M 2008 Annu. Rev. Phys. Chem. 59 233-59

[9] Davydov A 1977 J. Theor. Biol. 66 379-87

[10] Peyrard M 1995 Nonlinear Excitations in Biomolecules (Berlin: Springer)

[11] Dauxois T, Litvak-Hinenzon A, MacKay R and Spanoudaki A (eds) 2004 Energy Localisation and Transfer in Crystals, Biomolecules and Josephson Arrays (Advanced Series in Nonlinear Dynamics vol 22) (Singapore: World Scientific)

[12] Yu X and Leitner D 2003 J. Phys. Chem. B 107 1698-707

[13] Woutersen S and Hamm P 2002 J. Phys.: Condens. Matter 14 R1035-62

[14] Xie A, van der Meer L, Hoff W and Austin R H 2000 Phys. Rev. Lett. 84 5435-8

[15] Xie A, van der Meer A F G and Austin R H 2001 Phys. Rev. Lett. 88 18102-5

[16] Columbus F (ed) 2005 Soft Condensed Matter. New Research (New York: Nova)

[17] d'Ovidio F, Bohr H G and Lindgård P A 2003 J. Phys.: Condens. Matter 15 S1699-707

[18] Henzler-Wildman K A, Lei M, Thai V, Kerns S J, Karplus M and Kern D 2007 Nature 450 913-6

[19] Mingaleev S F, Christiansen P L, Gaididei Y B, Johansson M and Rasmussen K Ø 1999 J. Biol. Phys. 25 41-63

[20] d'Ovidio F, Bohr H G and Lindgard P A 2005 Phys. Rev. E 71026606

[21] Kopidakis G, Aubry S and Tsironis G P 2001 Phys. Rev. Lett. 87165501

[22] Archilla J F R, Gaididei Y B, Christiansen P L and Cuevas J 2002 J. Phys. A: Math. Gen. 35 8885-902

[23] Flach S and Gorbach A V 2008 Phys. Rep. 467 1-116

[24] Sato M and Sievers A 2009 J. Biol. Phys. 35 57-72

[25] Aubry S 2006 Phys. D: Nonlinear Phenom. 216 1-30

[26] Rumpf B 2007 Eur. Phys. Lett. 7826001

[27] Peyrard M 1998 Phys. D: Nonlinear Phenom. 119 184-99

[28] Flach S and Mutschke G 1994 Phys. Rev. E 49 5018-24

[29] Burlakov V M, Kiselev S A and Pyrkov V N 1990 Phys. Rev. B 42 4921-7
[30] Abdullaev F, Bang O and Sorensen M P (eds) 2001 Nonlinearity and Disorder: Theory and Applications vol 45 (Dordrecht: Kluwer)

[31] Rasmussen K O, Cai D, Bishop A R and Gronbech-Jensen N 1999 Europhys. Lett. 47 421-7

[32] Flach S and Willis C R 1998 Phys. Rep. 295 181-264

[33] Juanico B, Sanejouand Y H, Piazza F and De Los Rios P 2007 Phys. Rev. Lett. 99238104

[34] Livi R, Franzosi R and Oppo G L 2006 Phys. Rev. Lett. 97060401

[35] Piazza F, Lepri S and Livi R 2003 Chaos 13 637-45

[36] Reigada R, Sarmiento A and Lindenberg K 2001 Phys. Rev. E 6466608

[37] Dauxois T, Khomeriki R, Piazza F and Ruffo S 2005 Chaos 15015110

[38] Cretegny T, Dauxois T, Ruffo S and Torcini A 1998 Phys. D: Nonlinear Phenom. 121 109-26

[39] Piazza F and Sanejouand Y H 2008 Phys. Biol. 5026001

[40] Sitnitsky A 2006 Phys. A: Stat. Mech. Appl. 371 481-91

[41] Tirion M 1996 Phys. Rev. Lett. 77 1905-8

[42] Bahar I, Atilgan A R and Erman B 1997 Fold. Des. 2 173-81

[43] Hinsen K 1998 Proteins 33 417-29

[44] Micheletti C, Lattanzi G and Maritan A 2002 J. Mol. Biol. 321 909-21

[45] Kondrashov D, Van Wynsberghe A, Bannen R, Cui Q and Phillips G 2007 Structure 15 169-77

[46] Tama F and Sanejouand Y H 2001 Protein Eng. Des. Selection $141-6$

[47] Delarue M and Sanejouand Y H 2002 J. Mol. Biol. 320 1011-24

[48] Krebs W G, Alexandrov V, Wilson C A, Echols N, Yu H and Gerstein M 2002 Proteins 48 682-95

[49] Lu M and Ma J 2005 Biophys. J. 89 2395-401

[50] Nicolay S and Sanejouand Y H 2006 Phys. Rev. Lett. 96078104

[51] McCammon J A, Gelin B R, Karplus M and Wolynes P 1976 Nature 262 325-6

[52] Marques O and Sanejouand Y H 1995 Proteins 23 557-60

[53] Perahia D and Mouawad L 1995 Comput. Chem. 19 241-6

[54] Bahar I and Cui Q (eds) 2005 Normal Mode Analysis: Theory and Applications to Biological and Chemical Systems (C\&H/CRC Mathematical \& Computational Biology Series vol 9) (Boca Raton, FL: CRC Press)

[55] Tama F, Miyashita O and Brooks C L III 2004 J. Mol. Biol. 337 985-99

[56] Delarue M and Dumas P 2004 Proc. Natl Acad. Sci. USA 101 6957-62

[57] Suhre K and Sanejouand Y H 2004 Act. Cryst. D 60 796-9

[58] Brooks B and Karplus M 1983 Proc. Natl Acad. Sci. USA 80 6571-5

[59] Krimm S and Bandekar J 1986 Adv. Protein Chem. 38 181-364

[60] Yang L and Bahar I 2005 Structure 13 893-904

[61] Sacquin-Mora S, Laforet E and Lavery R 2007 Proteins 67 350-9

[62] Haliloglu T and Erman B 2009 Phys. Rev. Lett. 102088103

[63] Yan E, Kazmi M, Ganim Z, Hou J, Pan D, Chang B, Sakmar T and Mathies R 2003 Proc. Natl Acad. Sci. USA $1009262-7$

[64] Lockless S W and Ranganathan R 1999 Science 286 295-9

[65] Suel G M, Lockless S W, Wall M A and Ranganathan R 2003 Nat. Struct. Mol. Biol. 10 59-69

[66] Pisliakov A, Cao J, Kamerlin S and Warshel A 2009 Proc. Natl Acad. Sci. 106 17359-64 